\title{
Investigation of demagnetization in HTS stacked tapes implemented in electric machines as a result of crossed magnetic field
}

\author{
M. Baghdadi, H. S. Ruiz, J. F. Fagnard, M. Zhang, W. Wang, and T. A. Coombs
}

\begin{abstract}
This paper investigates the practical effectiveness of employing superconducting stacked tapes to superconducting electric machinery. The use of superconducting bulks in various practical applications has been addressed extensively in the literature. However, in practice, dramatic decrease in magnetization would occur on superconducting bulks due to the crossed field effect. In our study, we employed the superconducting stacked tapes in a synchronous superconducting motor, which was designed and fabricated in our laboratory, aiming to lessen demagnetization due to crossed field effect in comparison with superconducting bulks. Applying the transverse AC field, the effects of frequency, amplitude, and number of cycles of the transverse magnetic field are discussed. Furthermore, a stack of 16 layers of superconducting tapes is modelled and the consequences of applying the crossed magnetic field on the sample are evaluated. The confrontation between experiments and simulation allows us to thoroughly understand the crossed field effects on stacked tapes. At the end, a preventive treatment, based on the shielding characteristic of superconductor and materials with high permeability, i.e. $\mu$-metal and metalic glass, is suggested. On the other hand, the shielding feature of aforementioned materials will hinder the penetration of magnetic field and, consequently, reduction of the demagnetization will be attained.
\end{abstract}

Index Terms - demagnetization, superconducting motor, superconducting stacked tapes, transverse magnetic field.

\section{INTRODUCTION}

$\mathbf{H}$ IGH temperature superconductors have a good reputation of trapping high magnetic fields[1], [2] for practical applications where high magnetic field is required. In this regards, superconducting bulks have been used extensively in practice as a replacement for rare-earth permanent magnet [3]. However, regardless of practical challenges due to mechanical instability in high field imposed by Lorentz forces and low current density comparable the size of a bulk [4], [5], the crossed magnetic field has a destructive effect on the magnetization of superconducting bulks[6]. On the other hand, applying AC magnetic field on superconducting bulks would redistribute the initial current distribution inside the superconductor which leads to reduction of the main initial trapped field. The most severe demagnetization is when the AC magnetic field is perpendicular to the initial magnetization [6], [7], [8]. Thus, in

Manuscript received August 10, 2014.

M. Baghdadi, H. S. Ruiz, M. Zhang, W. Wang, and T. A. Coombs are with the Electric Engineering Division, Department of Engineering, University of Cambridge, Cambridge CB3 0FA, U.K. (e-mail: mb876@cam.ac.uk)

J. F. Fagnard is with the Electric Engineering Division, Department of Engineering, University of Cambridge, Cambridge CB3 OFA, U.K., and also with the Department of Electrical Engineering and Computer Science B28, Sart-Tilman, B-4000 Liège, Belgium

Color versions of one or more of the figures in this paper are available online at http://ieeexplore.ieee.org. order to validate the practical applicability of superconductors in order to be implemented as permanent magnets, it is a compelling urge to eliminate the demagnetization due to the crossed field effect.

The superconducting stacked tapes with a great potential to highly trap magnetic field was introduced in [9]. On the other hand, the sample, including two superconducting stacked tapes each with 120 layers, was able to trap field over 7 $\mathrm{T}$ at the temperature of $4.2 \mathrm{~K}$. This striking high value for the trapped magnetic field given the small size of the stack together with the commercial availability of the tapes, almost uniform physical properties in long lengths, and relatively higher mechanical strength compared with superconducting bulks [10], makes the stacked configuration an ideal candidate for strong magnet applications.

After successfully manufacturing a synchronous superconducting motor based on superconducting bulks [11], we designed and fabricated the second superconducting motor (Fig. 1(a)), in which the superconducting stacked tapes are implemented.

As shown in Fig. 1(b), superconducting tapes with the width of $46 \mathrm{~mm}$ were cut in the length of $76 \mathrm{~mm}$. Then they were stacked and placed inside the rotor that is integrated with the copper coil, which is used to initially magnetize the superconductors. In practice, using superconductors in this configuration, the HTS stack will experience magnetic field not only parallel to the initial magnetization, but also in every other angle. This work presents extreme case of demagnetization which is due to the crossed magnetic field.

Although the behaviour of type-II superconductors is well understood for the case where current density, $\mathbf{J}$, is perpendicular to magnetic induction field, $\mathbf{B}$, the theories for the case where $\mathbf{J}$ has components both perpendicular and parallel to $\mathbf{B}$ is still in a state of development. Several theoretical studies have been made in the literature aiming to describe the magnetic behaviour of irreversible type-II superconductors subjected to crossed magnetic fields including generalized double critical-state model, Brandt and Mikitik model, original elliptic critical-state model, extended elliptic critical-state model and Badia, Lopez, and Ruiz model [12], [13].

The objective of this work is to investigate the effects of crossed magnetic field on the magnetization of superconducting stacked tapes through varying the amplitude, frequency, and the number of cycles of transverse magnetic field. Furthermore, a 2-D model of stacked tapes based on finite element method is employed to ascertain the validity of experimental results. Finally, a protective method is proposed in order to decrease the demagnetization both on superconducting bulks 

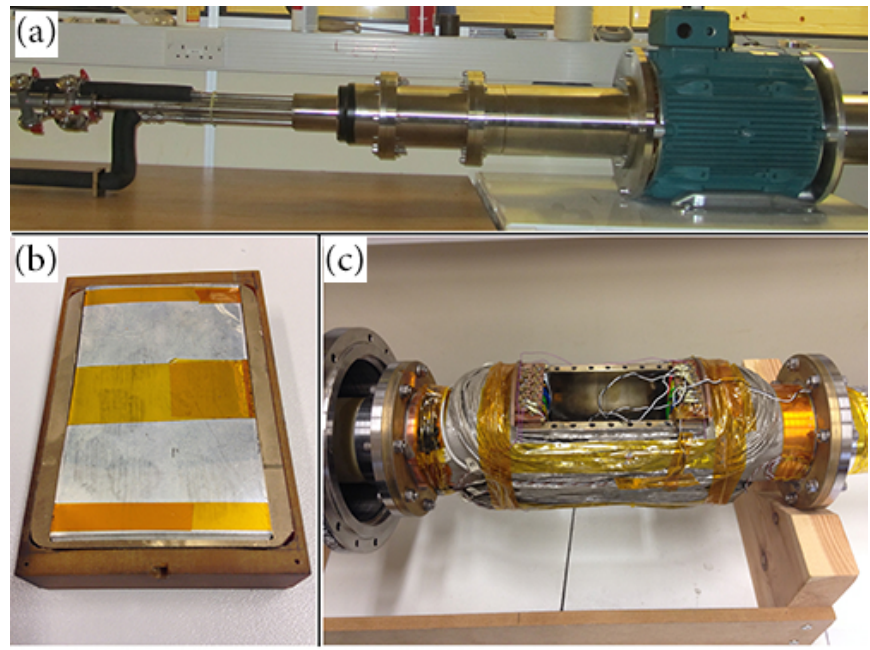

Fig. 1. (Color online) The superconducting synchronous motor based on HTS stacked tapes (a). The superconducting stacked tapes are placed in the superconducting cassette (b). The rotor is shown in (c).

and stacked tapes.

\section{EXPERIMENTAL SETUP}

The sample consists of a stack of sixteen $12 \mathrm{~mm}$ square of HTS superconductors from SuperPower Inc.. The overview of the experimental rig is depicted in Fig. 2(a). The sample is then placed in a 3-D printed nylon cassette where two linear arrays of Hall probe boards are located on beneath and one side of the holder (Fig. 2(b)). The first Hall probe board array, shown in red, has 5 Hall probes located under the bottom layer of the stacked tapes. These sensors are implemented to measure not only the initial trapped magnetic field, where there is no transverse AC field, $\mu_{0} H_{a b}^{\text {peak }}=0 \mathrm{~T}$, in the sample due to perpendicular applied magnetic field in respect to the wider surface of the superconductors, $H_{\perp c}$, but also the decay of magnetizations due to transverse AC magnetic field, $H_{\| c}$. The second Hall probe board, shown in green, includes two Hall probes which are responsible of the measurement of $H_{\| c}$. The cassette is then placed inside a nylon rod. Both rod and the holder are then located inside a cylindrical nitrogen bath made of a polycarbonate tube that is attached from the top to a nitrogen reservoir. Having several materials with different expansion rates, we employed finite element analysis to be confident that the experimental rig can be safely used at low temperature, $77 \mathrm{~K}$.

In all the experiments, the wider surface of the stack is initially placed parallel with poles of the magnet and are magnetized using field cooled magnetization. The amplitude of the magnetic field, $H_{\| c}$, in field cooled magnetization was set relatively high to be confident the sample will be fully magnetized, i.e. $119 \mathrm{mT}$, in order to have a same baseline for all experiments. Following the removal of background field, a constant time interval, i.e. 600 seconds, was then employed in order to allow the trapped magnetic field to relax due to thermally activated flux creep. Getting ready for applying the crossed field, the sample is turned 90 degrees, thus, the direction of the magnetic field would be in parallel (a)

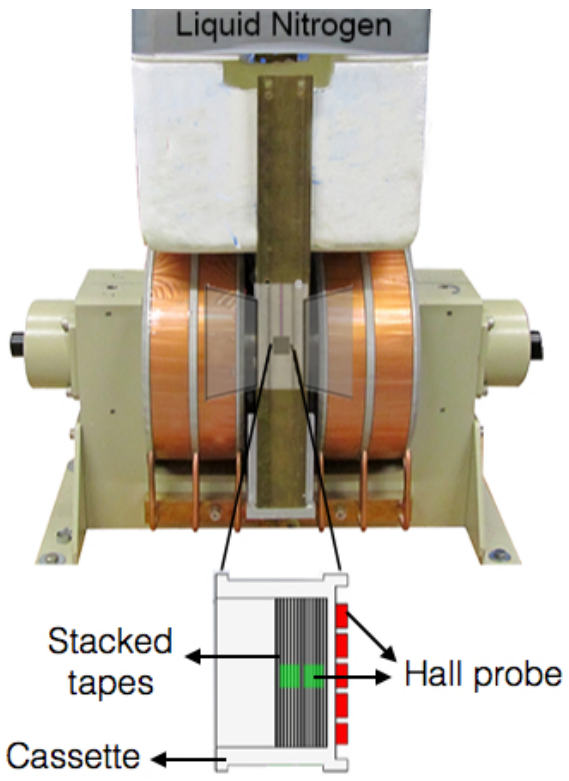

Fig. 2. (Color online) The experimental rig is shown in (a). The cassette containing superconducting stacked tapes and Hall probe boards are depicted in (b).

to wider surface of the superconductor. Considering various amplitude of transverse magnetic fields, the experiments could be conducted either at a constant sweep rate $\left(\frac{d B}{d t}=\right.$ const $)$ or a fixed frequency ( $f=$ const). In our study we used the latter one in which frequencies $0.5 \mathrm{~Hz}, 2.5 \mathrm{~Hz}$, and $7.5 \mathrm{~Hz}$ with different amplitudes, $\left|\mu_{0} H_{a b}^{\text {peak }}\right|=80 \mathrm{mT}, 150 \mathrm{mT}, 220$, $\mathrm{mT}$, and $300 \mathrm{mT}$ were applied. Furthermore, each experiment is conducted for 100 cycles in order to observe the consequences of applying several cycles of AC transverse field on demagnetization.

\section{RESULTS AND DISCUSSION}

An exemplary graph of linear reduction of the magnetization for a AC transverse field with the amplitude of $\mu_{0} H_{a b}^{\text {peak }}=80$ $\mathrm{mT}$ and the frequency of $0.5 \mathrm{~Hz}$ for 100 cycles is given in Fig. 3. In the first look it is noticeable that the effect of the crossed

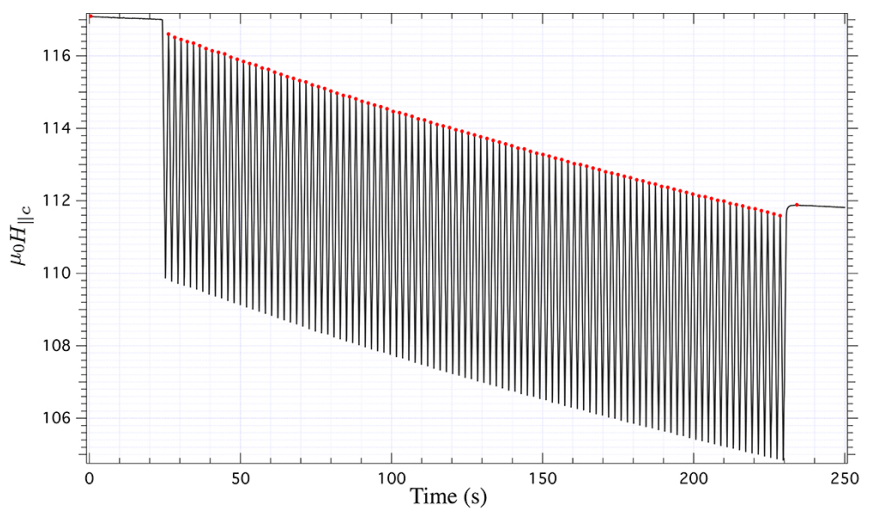

Fig. 3. (Color online) The trajectory of the demagnetization for 100 cycles while a transverse AC field of $\mu_{0} H_{a b}^{\text {peak }}=80 \mathrm{mT}$ with the frequency of $0.5 \mathrm{~Hz}$. is applied. The peaks are depicted in red. 
field on the magnetization is slight. On the other hand, there is no sign of a dramatic plunge in magnetization as was reported for superconducting bulks [6]. In our case, having the linear pattern for demagnetization, the decrement of magnetization for one cycle is very small.

The maximum percentage of demagnetization for various amplitude and operating frequencies of transverse magnetic field $H_{a b}$ are given in Fig. 4. Furthermore, demagnetization in the stacked tapes varies inversely with frequency under the same applied transverse magnetic field.

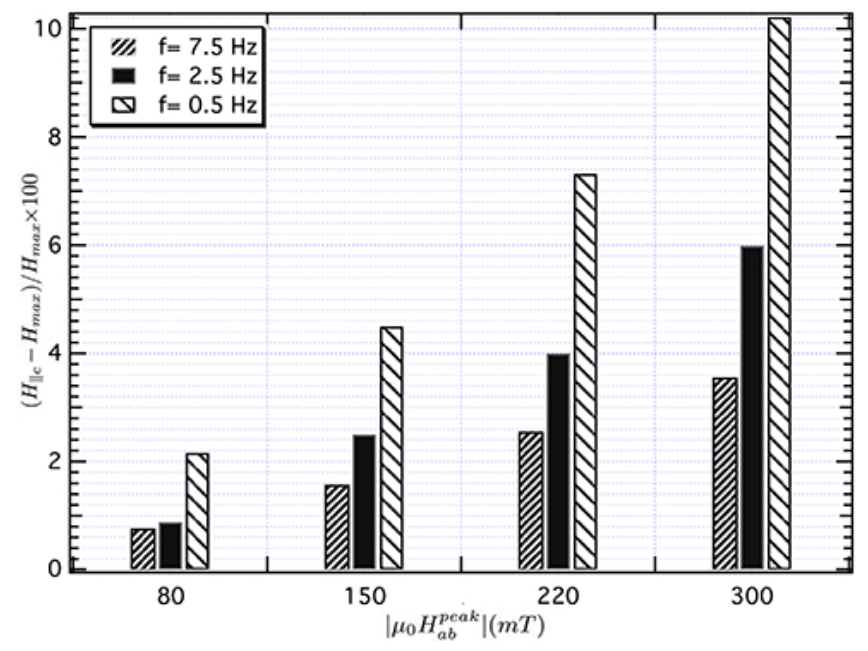

Fig. 4. The percentage of demagnetization, $\left(H_{\| c}-H_{\max }\right) / H_{\max } \times 100$, for different amplitudes and frequencies of the applied AC transverse magnetic field $H_{a b}$.

Comparing with studies carried on superconducting bulks[6] in which more than $50 \%$ of the initial trapped field was lost after just one cycle for $H_{a b} / H_{\| c} \approx 1.5$, the demagnetization factor of the stacked tapes is strikingly lower, $\sim 10 \%$ after 100 cycles for $H_{a b} / H_{\| c} \approx 3$ ). This achievement in reduction of the demagnetization makes the stacked configuration very attractive for practical applications.

The reason for this impressive reduction can be explained by comparing the aspect ratio of superconducting bulks with stacked tapes. On the other hand, the aspect ratio of the superconducting bulk in [6] was 4 while in stacked tapes is $\frac{12 \mathrm{~mm}}{1 \mu \mathrm{m}}=12000$. Having high aspect ratio, because of very low thickness of superconducting tapes, increases the amplitude of minimum critical Lorentz force required for removing the pinned vortices from pinning centres [14], that consequently leads to smaller demagnetization on stacked tapes comparing with bulks.

This phenomenon can also be explained by observing the significant increment of relaxation time in superconductors due to transverse field in superconducting stacked tapes as it is inversely proportional with the thickness of a sample, $\dot{t}=(a / c) \pi J_{c \perp} /\left(\omega H_{a b}^{\text {peak }}\right)$ [14] where $t$ is relaxation time, $c$ is the thickness, $\omega$ is the frequency, and $a$ is the length of the sample. Thus, when the field $H_{a b}$ is not too large, i.e., $\mu_{0} H \perp_{c}<J_{c \perp} a$, the sheet current $\left(J_{x}, J_{y}\right)$ decays very little during a cycle of the AC field and therefore, the relaxation time increases as the thickness of the sample decreases.
To validate the experimental results, we model the crossed field effect using finite element analysis. The governing equations, derived from Maxwell's equations, are given in (1) and (2):

$$
\begin{array}{r}
\nabla \times(\rho \nabla \times \mathbf{H})=-\mu \frac{\partial \mathbf{H}}{\partial t} \\
\nabla \times \mathbf{H}=\mathbf{J}
\end{array}
$$

where $\mathbf{H}=\left[H_{x}, H_{y}, H_{z}\right]^{T}, \mathbf{J}=\left[J_{x}, J_{y}, J_{z}\right]^{T}, \rho=$ $\frac{E_{c}}{J_{c}}\left(\frac{|\mathrm{J}|}{J_{c}}\right)^{n-1}$, and $J_{c}$ is defined as the current at which $E_{0}=$ $1 \times 10^{-4} V m^{-1}$ is reached under DC $I-V$ measurements. Besides, the electrical behavior of superconductor is modeled by $E-J$ power law:

$$
\mathbf{E}=\rho \mathbf{J}
$$

where $\mathbf{E}=\left[E_{x}, E_{y}, E_{z}\right]^{T}$. The partial differential equations will be then extracted from Maxwell equations and be solved by finite element analysis.

It should be noted that, the thickness of the sample is crucial while simulating the crossed field effect. However, putting the actual thickness for each layer, $1 \mu \mathrm{m}$, will increase the degrees of freedom (DOF) dramatically that, consequently, leads to relatively high computation time. Therefore, we set the thickness to $100 \mu \mathrm{m}$ to satisfy the low thickness comparing with the wider surface, which is $12 \mathrm{~mm}$ and also to keep the DOF in a sensible range.

The distribution of the current densities of initial field cooled magnetization and after applying transverse AC field, $\mu_{0} H_{a b}^{\text {peak }}=300 \mathrm{mT}$, and the frequency of $0.5 \mathrm{~Hz}$ are depicted in Fig. 5(a). It is evident that, regardless of slight changes in the middle of the sample, the overall pattern of current density in the sample is merely changed. On the other hand, comparing these results with the simulation results for a superconducting bulk [6], in which applying $\left(H_{a b} / H_{\| c} \approx 1.5\right)$ would lead to the total collapse of magnetization, the maximum reduction of magnetization in this simulation is $\simeq 8 \%$ (Fig. 5(b)), which is in a good agreement with the experiment.

As shown in Fig. 6, the use of superconducting tapes integrated with some materials with high relative permeability is proposed as a preventive method to shield transverse magnetic field. On the other hand, the superconducting tapes are highly competent to shield DC and AC magnetic field. [15], [16] The idea is to wrap several layers of superconducting tapes around the sample, e.g. HTS bulk or tape, in order to decrease the intensity of the magnet field experienced by the sample, and, conclusively, the rate of the demagnetization would be dropped. Similarly, we can use materials such as $\mu$-metal or metallic glass[17] as their high value of permeability provide a low reluctance path for magnetic flux and, subsequently, shield against static or slowly varying magnetic fields. It should be noted that $\mu$-metal is saturated at low magnetic field, therefore, using several layers is advised; each layer shields a fraction of magnetic field.

\section{CONCLUSION}

This paper addresses in details the demagnetization effect in a stack of superconducting tapes that was subjected various 
(a)

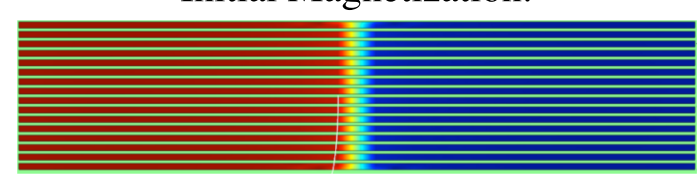

After applying transverse field:

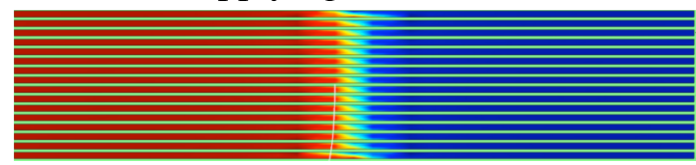

$1.4 \mathrm{E} 8$

(b)

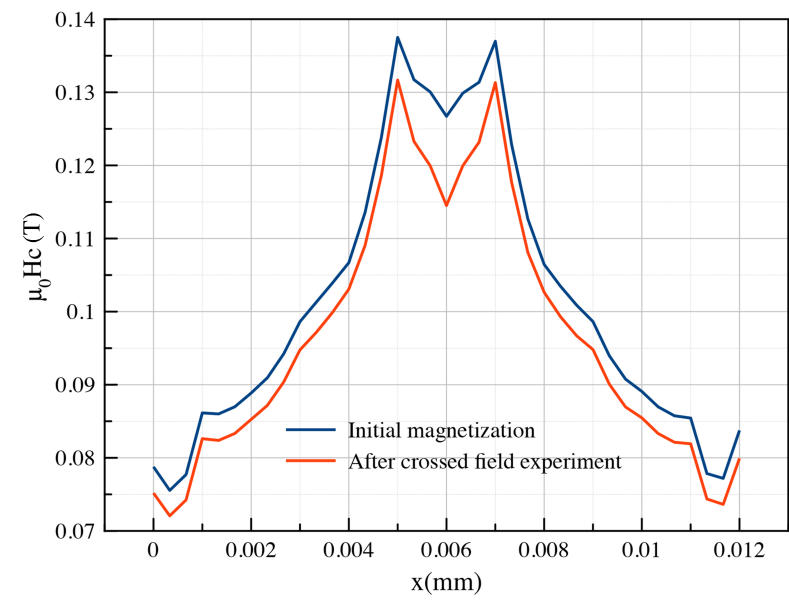

Fig. 5. (Color online) Simulation results: Current distribution (a) for the stacked tapes and magnetic flux denstiy (b) for before applying the AC transverse magnetic field, $\mu_{0} H_{a b}=0$ and after 100 cycles of $H_{a b}^{\text {peak }}=300$ $\mathrm{mT}$, for the operation frequency of $f=0.5 \mathrm{~Hz}$

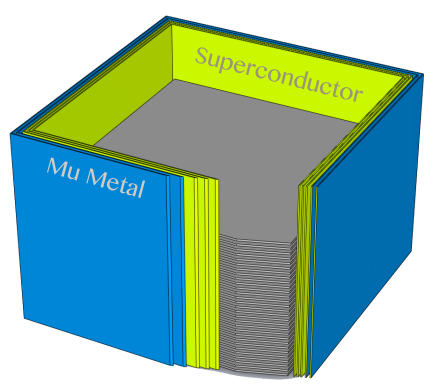

Fig. 6. (Color online) Preventive solution for decreasing the demagnetization due to crossed filed effect for both superconducting bulk and stacked tapes

amplitudes and frequencies of transverse magnetic fields. The maximum decrement of initial magnetization for the case where $H_{a b} / H_{\| c} \approx 2.5$ that was about $10 \%$ that makes the stacked tapes as a good substitution for superconducting bulks in superconducting motors. Furthermore, a 2-D model of HTS stacked tapes based on finite element method was employed and the results were in a good agreement with the experiment. Finally, in order to even make the superconductors more interesting for practical for real applications, we proposed a method to decrease the demagnetization by basically wrapping the HTS tapes or some materials with high permeability around the sample.

\section{ACKNOWLEDGMENT}

The authors would like to thank Prof. A. Campbell of the Department of Engineering, University of Cambridge, Cambridge, U.K., for his valuable advice on this work.

\section{REFERENCES}

[1] M. Tomita and M. Murakami, "High-temperature superconductor bulk magnets that can trap magnetic fields of over 17 tesla at 29 K", Nature, vol., 421, no. 6922, p. 517, Jan. 2003.

[2] G. Krabbes, G. Fuchs, P. verges, P. Diko, G. Stover, and S. Gruss, "16 T trapped fields in modified YBaCuO: materials aspects", Physica $C$, vol. 378 , no. 1, p. 636, Oct. 2002.

[3] M. Miki, S. Tokura, H. Hayakawa, H. Inami, M. Kitano, H. Matsuzaki, Y. Kimura, I. Ohtani, E. Morita, H. Ogata, M. Izumi, H. Sugimoto, and T. Ida,"Development of a synchronous motor with $\mathrm{GdBaCuO}$ bulk superconductors as pole-field magnets for propulsion system", Supercond. Sci. Technol., vol. 19, no.7, p. S494, Apr. 2006.

[4] C. K. McMichael, K. B. Ma, M. A. Lamb, M. W. Lin, L. Chow, R. L. Meng, P. H. Hor, and W. K. Chu, "Practical adaptation in bulk superconducting magnetic bearing", Appl. Phys. Lett., vol. 60, no. 15, p. 13, Feb. 1992.

[5] H. Fujimoto, "Technical issues of a high- $\mathrm{T}_{\mathrm{C}}$ superconducting bulk magnet", Supercond. Sci. Technol., vol. 13, no. 6, p. 827, Feb. 2000.

[6] P. Vanderbemden, Z. Hong, T. A. Coombs, M. Ausloos, N. Hari Babu, D. A. Cardwell, and A. M. Campbell, "Remagnetization of bulk hightemperature superconductors subjected to crossed and rotating magnetic fields", Supercond. Sci. Technol., vol. 20, no. 9, p. S174, Aug. 2007.

[7] Z. Hong; Y. Jiang, R. Pei, W. Yuan, R. Marchant, T. A. Coombs, "Numerical Analysis of the demagnetization effect in a superconducting machine with bulk HTS material on the rotor", IEEE Trans. Appl. Supercond., vol. 19, no.3, p. 2897, Jun. 2009.

[8] M. Baghdadi, H.S. Ruiz; T. A. Coombs, "Crossed-magnetic-field experiments on stacked second generation superconducting tapes: Reduction of the demagnetization effects", Appl. Phys. Lett., vol. 104, no. 23, p. 232602, Jun. 2014

[9] A. Patel, K. Filar, V. I. Nizhankovskii, S. C. Hopkins, and B. A. Glowacki, "Trapped fields greater than $7 \mathrm{~T}$ in a $12 \mathrm{~mm}$ square stack of commercial high-temperature superconducting tape", Appl. Phys. Lett., vol. 102, no. 10, p. 102601, Mar. 2013.

[10] K. Selva and G. Majkic, "Trapped magnetic field profiles of arrays of $(\mathrm{Gd}, \mathrm{Y}) \mathrm{Ba} 2 \mathrm{Cu} 3 \mathrm{Ox}$ superconductor tape in different stacking configurations", Supercond. Sci. Technol., vol. 26, no. 11, p. 115006, Oct. 2012.

[11] Z. Huang, W. Xian, M. Zhang, M. Chudy, Y. Chen, Z. Zhong, M. Baghdadi, W. Wang, F. Spaven, K. Matsuda, and T. A. Coombs, "Control and operation of a high temperature superconducting synchronous motor", IEEE Trans. Appl. Supercond., vol. 23, no. 3, p. 5200204, Jun. 2013.

[12] E. H. Brandt, "Superconductors of finite thickness in a perpendicular magnetic field: Strips and slabs", Phys. Rev. B, vol., 50, no. 6, p. 4246, Aug. 1996.

[13] J. R. Clem, M. Weigand, J. H. Durrell, and A. M. Campbell, "Theory and experiment testing flux-line cutting physics", Supercond. Sci. Technol., vol. 24 , no. 6 , p. 062002 , Mar. 2011.

[14] G. P. Mikitik and E. H. Brandt, "Vortex shaking in rectangular superconducting platelets", Phys. Rev. B, vol. 69, no. 13, p. 134521, Apr. 2004.

[15] J. F. Fagnard, M. Dirickx, G. A. Levin, P. N. Barnes, B. Vanderheyden, P. Vanderbemden, "Use of second generation coated conductors for efficient shielding of dc magnetic fields", J. Appl. Phys., vol. 108, no. 1, p. 013910, Jul. 2010.

[16] J. Kvitkovic, S Pamidi, and J. Voccio, "Shielding AC magnetic fields using commercial $\mathrm{YBa}_{2} \mathrm{Cu}_{3} \mathrm{O}_{7}$-coated conductor tapes", Supercond. Sci. Technol., vol. 22, no. 12, p. 125009, Oct. 2009.

[17] V. Serban, C. Codrean, and D. Utu and A. Ercuta, "Fe-based bulk metallic glasses used for magnetic shielding", Journal of Physics: Conference Series, vol. 144, no. 1, p. 012037, 2009. 